\title{
A NEW EXPLANATION FOR FLARES ON dMe STARS
}

\author{
G.M.Simnet t \\ Department of Space Research, University of Birninghanu, B15 2TT, England
}

\begin{abstract}
It has been propesed that non-thermal ions dominate the energy transfer at the onset of sular flares. Here we exannine this hypothesis in the context of flares on dMe stars. If the magnetic field in the stellar corona is significantly larger than that in the solar corona, and if strong ficlds in the photosphere, analingons to active regions, are absent, then a self-consistent explanation of stellar flares may be formulated.
\end{abstract}

\section{Intrualuction}

Understinding flares in flare stars is related intinate!y to understanding the energy transport from the: mitgnetic field to the lower atmosphere. The only mechanisus considered by Ilatisch (1986) in his commulus it solar-stellar flares were thick-target interactions by non-thermal electron beams, or thernat conduction. Wi believe another process is worth investigating. Sinnett (1986) and Martens (1058) proposed thint in transient conergy releases in the corona the energy transfer mediun from the mannetic field to the plasmat is it neutral ion bean in which essentially all the energy resides in the ions (protonsi). With thisi concept the ions predoninantly heat the atmosphere, which would manifest itself in enhanced U-band anission. The production of X-rays is incidental to this heating as the ions normally deposit most of their energy via Conlonl) cullisions. In the Sun the sudden density increase at the transition region, conpled with the colum deptl of the transition region from the corona, produces conditions appropriate for the generation of the $X$-ray bur:it. In dNe stars the physical couditions are different and the $X$-ray signatures are anditively weiker than in the Sun.

\section{The Important Properties of dMe Flare Stal's}

Properties of dMe stars that are relevant to this discussion are: the optical flare light curve; rapid rotation; strong coronal magnetic fields; and large-scate coronal magnetic loops. The U-baud light curve of a

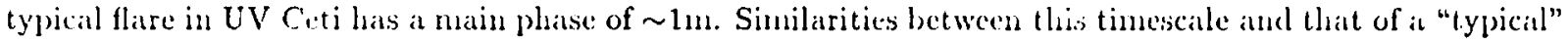
solar flare harel X-ray burst are apparent. We interpret the inpolsive part of both as representative of the duration of energy deposition in the chromosphere; coronal heating may be over a much longer period.

The hot gas in the X-ray-enitting coronae of nearby dMe stars is contained by the coronal nagnetic field and Rosner et al., (1985) recognized in the solar case that this magnetic field is central both to quiescent $X$-ray emission and to flares. The generation of the magnetic field is attributed to a dymano mechanism, sustained by having a convection zone in a rotating star. For stars of spectral type latter than F, which hatve a convection zone, the quiescent $X$-ray luminosity $\propto$ (rotation rate) ${ }^{2}$ (Rosner et al., 1985). For stars litter than $\mathrm{M} 5.5, \sim 100 \%$ of $\mathrm{M}$ dwarfs are dMfe stars; they are generally snatl stars $\left(0.1 \mathrm{M}_{\odot}\right)$ and are probibly fully convective. Thus we expect rotating dale stars to have stron! coronal fields. Gianupapat and Rosner (108-1) showed that stars with shallow convection zones, such as the Sun, should exhibit a significant reduction in the size of a typical active region. Such stars will have strong X-raty flares, compared with the magnitude of the energy release, as the snall-scale field concentrates the energy flux better. Conversely, there will be lower quiescent X-ray emission as the large-scale coronal firlds are lower, and can contain a lower matter densily, which results in an overall low enission measmere. The magnetic fick strughth is an inportant parander, ats it is the flare energy souree and also restrains the accelerated ions such that they lose their energy in the: stellar atmosplisere. Strong solar fields, $\geq 0.1 \mathrm{~T}$, only exist, above the convection zone, in active regions, and coronal fields are very weak by comparison. On flare stars Mullan (1976) suggested IT for an event from BY Dra, but more recently, estinates of the magnetic fields needed to account for microwave conission have been made which are smaller, ranging from 0.025T to 0.3T (Gary et al., 1982; Kundu and Shevganukiar, 19S5; Dastian and Bookbinder, 1987). 


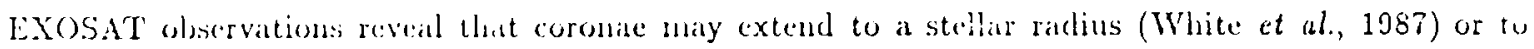
suveral stellatr radii (Collier Cameron et al., 1988). VLBI measurements of Algol (Hjellning, 1980) indicate

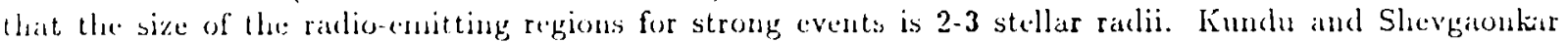
(1985) infire sizes even liarger, up to 6 stellar radii for Y'Z CMi. Occasionally loops extending to severall $R_{\sigma}$ are meded to arcenut for solar phenomena (Leblanc and Hoyos, 1985). There is no evilince that such loops are permanent, althongh thay have bern inferred to exisc lior as long as 5 days (Sinnett and Holt, 1971).

aMe stars have high gluicsent X-ray luninosity, typically $>>10^{28} \mathrm{erg} \mathrm{s}^{-1}$ compared with the solar luninesity of $<10^{27}$ "rg $\mathrm{s}^{-1}$. As the effective $\mathrm{X}$-raty temuperatures are in the $10^{6} \geq 10^{7} \mathrm{~K}$ ramge then the incrasel luninesity, over the solar value, must be mainly due to an increased emission measure $\left(\mathrm{n}_{e}^{2} \mathrm{~V}\right)$. Thus rither the coronal lespes in flare stars are very large, or the curonal densities are high, or both.

In sumbuaty, it is evident that conditions on flare stars involve large coronal structures, with high

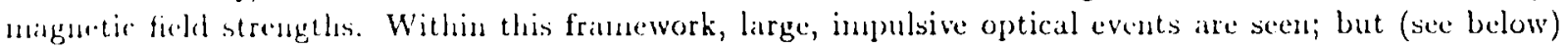
the soft $X$ ray erents that are sonietines olserved in coincidence tend to be more slowly varying.

\section{Ion Acceleration}

Fumblamental to our hypothesis is the requirenent that ion acceleration be a matural conseguence of natprelic energy relcatse. A likely acceleration mechaniss is a magnetosonic slock, although as Martens (I9S8) hats shown, direct electric field acceleration may atso be sulisfactory. In his review of shock format tion and evolution in the soliar atmosphere, Bougeret. (1985) emphasized that shocks "are anong the most

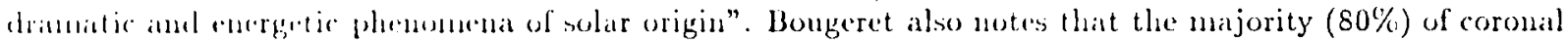
type Il rantio burste, which are ciused by strong shocks moving up) through the corona, are associated with sub flates or alass 1 Hares; therefore they are not an indication of a big flare.

'Tharectically shock acceleration has been studied extensively (eg. Decker and Vlahos, 19S5; Ohsawa and Sitkit, 1987). (Ohsawa and Sikail show that ions are readily accelerated up to a velocity:

$$
v \sim v_{A}\left(m_{i} / m_{c}\right)^{1 / 2}\left(M_{A}-1\right)^{3 / 2}
$$

where ${ }^{A}$ is the Alfven velocity and $M_{A}$ is the Alfven Mach mumber. For an Alfvén velocity of $400 \mathrm{~km} \mathrm{~s}^{-1}$ and $M_{1}=3$, the proton cocrg'y is around $10 \mathrm{MeV}$. Electron acceleration in high-Mach-munber shocks has leedn arderiessed by Tukat et al., (1986) but they point ont that even under the most favourable conditions it is difficult to put mone than about $1 \%$ of the released energy into the electrons. Thus our conclusion is that inn accoldration is likely to dominate during any realistic shock acceleration prociess in the stellar context.

'There are many examples of proton acceleration within the solar system, e.g.:

1. In the MeV region of the spectrmu, high proton fluxes of solar origin are extremely conmon in the interplunetary medium. There is a solar cycle dependence and the flux is higher at solar maxinum than at solar mininum. Around the last solar maximum, July December, 1979, the $0.97-1.85 \mathrm{MTeV}$ proton int ensity wats 210 protons $\mathrm{cm}^{-2} \mathrm{~s}^{-1} \mathrm{sr}^{-1} \mathrm{MeV}^{-1}$ for 47.6 days out of 18.4 . The largest "flare" associated "vents excend this flux hy over three orders of magnitude. By comparison, the lowest intensities recorded were $<10^{-2}$ protuns $\mathrm{cm}^{-2} \mathrm{~s}^{-1} \mathrm{sr}^{-1} \mathrm{McV}^{-1}$, and this level was experienced on only a few days in this preriod. This illustrates the ease with which the Sun produces such particles. If we take 10 protons $\mathrm{cm}^{-2}$ $\mathrm{s}^{-1} \mathrm{sr}^{-1} \mathrm{Ml} \cdot \mathrm{V}^{-1}$ ats typical of the coronal leakinge, and assume that it is emitted isotropically with a spectrmu such ats that meatsured by Simalujat ct al., 1983, then the energy flux in such particles is $\sim 10^{24}$ erg $s^{-1}$. This is culy around three orders of magnitude less than the quiet time solar X-ray luminosity. The difference between the interplanetary proton flux $\sim 1 \mathrm{MeV}$ during quiet times and extrene flare pribels is $\sim 10^{6}$. If energy release is occurring continuonsly via matgnetic reconnection in the corona, then there will be contimuns acceleration. The energy given to the protons must re-appear sonewhere. It maty be retained, but re-distributed, in the corona, thereby heating it; it may be transported and chunped in the chromosphere, thereby producing a "Hlare"; or it may be released into space. It is not ditficult to inninge that the magnetic fiedd plays an inportant role in controlling the destiny of this energy. What fraction of accelerated protons are detected in interplanetary space is an open question, and it is egually open as to the contribution such particlis nuake to coronal heating. Energy deposition ly accelerated ims is a platusille candidate for re-clistriluting the energy releascel by the coronal fiedd into global lieating of the coronal gats. 
2. Sonne large low-energy (<2 MeV) proton events are absociated only with disappearing filanuents (e.g. Sanahuja et al., 198j). We interpret these as events where the topology of the coronal tield was inappropriate to contain the accelerated ions, so instead of producing a flare" they escaped into space.

3. Trapped protons in the Jovian magnetosphere observed by spacecraft have a spectrum which flattens around $1 \mathrm{MeV}$ (McDonald et al., 1979). The bulk of the energy in such a spectrum is $\sim 0.11 \mathrm{MeV}$.

In smmmary, theoretical results have demonstrated the ease with which ions may be accelerated; this is not the case with electrons. The accelerated energy spectrum is a function of the shock parameters, but where direct observations can be made the bulk of the energy resides in protons in the $0.1 \quad 1$ MeV region.

\section{Relevance to Observations of Flares in dMe Stars}

Several flare observations of dMe stars cover more than one wavelength band. A strong (3.5 10 $10^{31}$ ergs), long-lived $\left(\sim 2 h_{h}\right) X$-raty flare from Proxima Centauri (Haisch et al., 1983) was observed bot hy binstein and IUE. The IUE spectrum at the peak of the X-ray event showed considerably enhanced enission lines. Thus this flare could be interpreted as a large solar-type flare. Flares from UV Ceti, and other dMle stars, tend to be such shorter. The first co-ordinated optical and X-ray olsservations of a flitre in UV Ceti (Heise at ab, 1975). showed that the optical light curve peaks earlier than the X-ray light eurve and decays faster. A 5n-duration flare in YZ CMi (Doyle et al., 1988) exhibited no correlated X-ray emission above the EXOSAT threshold; the only X-ray event close to the optical flare reached nuximan 11 ua lat re. The lack of correlated X-ray activity for this flare completely elininates any concept that the energy transfer is via electron beans.

We now exanuine how these observations are explained quite naturally by the following sconariu. Suppose that energy release in the corona is tranferred to accelerated ions which nove towards the chromesplere over a large areia. (In this context "large" is in comparison with the fraction of the solitr surface occupped by a solar active region. Recall that for stars with deep convection zones the sire of any active region would be large compared with the sular case and therefore the energy deposition is likely to be more diftuse in a dMe star than in the Sun.) This will intially result in heating; lence the dramatic increase in the U-band enission. In high density regions and with low energy flux the temperature rise jis offset by rapial cooling, so the plasma never reaches X-ray emitting temperatures. The heated material is driven into the stellan corona as an expanding, upwiad moving mass. Two things now happen; 1) the rising mass presents a high colmm density to ions in transit to the chromospluere from the corona; this colum can absorb the energy of any accelerated ions $(0.1 \mathrm{I} \mathrm{MeV})$ still being produced before reaching the chromosphere; this renuves the driver for the evaporation; 2) the energy absorbed in the rising column of plasma heats it. The difference, however, from the intial sitnation is that the lower density results in a much longer cooling time. l'rovided energy is still fod in the temperature will rise to a value which naty be high enough to entit $X$-ray's.

We believe this explains quite naturally why the soft $X$-ray flux peaks after the optical flux, or why in some catses, if the energy release stops prematurely, it may be entirely absent. Also, if a fresh energy release occurs before the evaporated material has drained bick to the base of the corona, the ligh density coronal bas may be heated to X-ray enitting tempuatures withont any noticeable effect in the chromosphere, which is presumed to be the primary site of the U-band Hux. The observations of de Jager ct al, (1986) of activity in BY Dra are consistent with our thesis; in this event the bulk of the soft $\mathrm{X}$-ray event occurred soune Gun after the peak in the U-band Hux.

We noted above that the quiescent $X$-ray lmminosity is relat:vely ligh in dMe flare stars. This is interpreted as heating due to Coulomb collinjuns of ions which are, presumably, quasi-continuously being accelerated by the shocks induced by magnetic reconnection in the stellar corona. Stellar flares occur when the magnetic field geometry changes slightly to allow direct acess of the accelerated ions to the chromosphere, or when the accelerator becones slightly more effective in producing energutic iuns and shifts the peak in the energy spectrm to higher energies, thereby allowing deeper penetration into the atmosphere. If the energy release is quasi-continuous, then the nean $X$-ray luminosity and the tinse-averaged flare energy are both likely to be correlated with the rate of energy release; it is merely dumperl in a different part of the atmosylure. This correlation lats been noted (Doyle and Butler, 1985).

There are relatively few co-ordinated radio observations of stellar flares. Pajors on radio bursts from Hare stars (e.g. Gary et al., 1982; Kundu et al., 1987) attribute inuch of the microwave muission to a coherent electron-cyclotron maser. There are, nevertheless, observations which do not secm to fall in this category (Bastian and Bookbinder, 1957). Although the inupulsive flares they observed were attributed to a colerent 


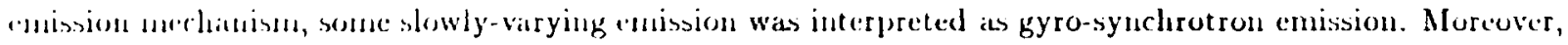

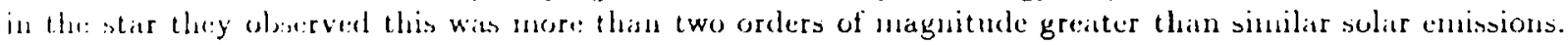
Thase clearly ment large mumbers of electrons. However, this is not in conflirt with our basic premise; we ale not advoraling that there are $n 0$ electrons accelerated in flare stars, merely that in terms of the energy buctger they atre insignificant.

\section{Predictions}

In ronclusion thene are screral predietions that are alderopriate to make.

1. Red shift: in the line spertra. If an ion heam travels downwards and is stopped, due to charge-exchange

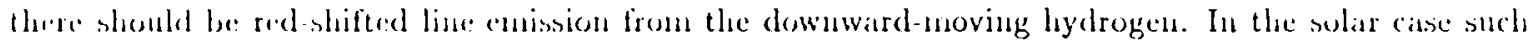

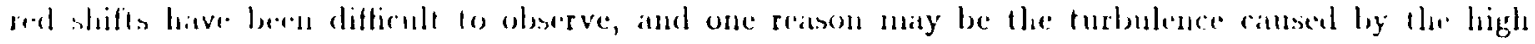

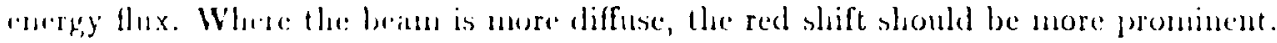

2. Ciamma ray enubsion is anticipated from a large stellar flare. A detector of 2500 cme semsitive area ogresaling in the 1 - 10 MeV range rlose to the capability of the Gamma Ray Observatory is on the

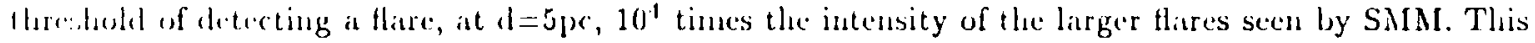
atsomuses lle defectability is photon limined, which is reasonable if the time and position of the flare are kuswa from observations at ot lar witelengths.

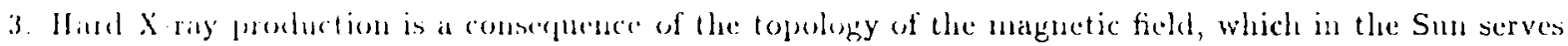

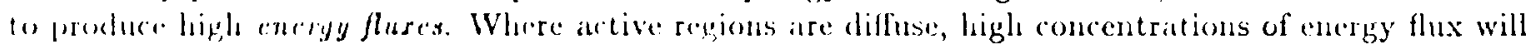
unt orent and hand X raty anission will be inhibited. IVe predict that only rirely will dMe stirr flares le olourved where the ratio of hard/soft X-rays is as high as in at "typical" solar flare.

1. Miencentwe radin bursts, which are associated with the production of hard X-rays, should also be latgely absent in alde stars. In the Sun lac microwave burst (which is mainly incolerent gyrosynchrotron ramlintien) comes from electrons which escape from the $X$-ray-enteting region; this is why microwave

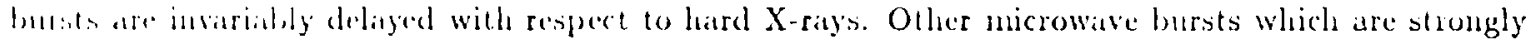
polatizal probably come from matser ation, and it is not appropriate for us to comment on these.

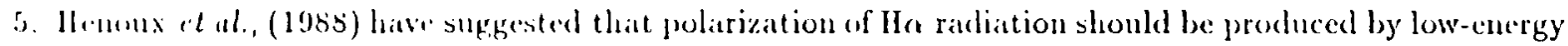

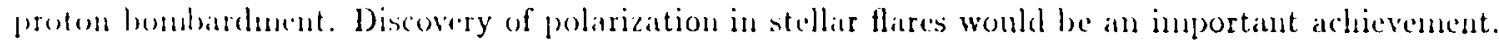

\section{i. References}

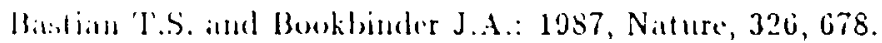

lHongerel J.1.: 1955, in "Collisimless Shocks in the Holiosphere", AGU Monograph, $35,1$.

(ollier Cameron A., Bulford D.K., Rucinsti s.M., Villu O). and White N.E.: 1988, MINRAS, 231.131.

Derher li.13. and Vlahws L.: 1955, J. Geopliys: Res.s, 90, 47.

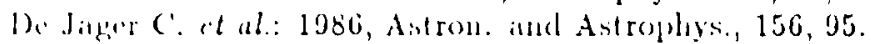

Boylu. J.C., Butler C.J., Byrne P.B. and van den Oord G.HI.J.: 1988, Astron. and Astrophys., $193,229$.

Buyle J.C. atul Hutler (:J.: 1035, Niture, 313, 378.

(iary D.E, I.iusky J.L. and Dulk G.A.: 1952, Astropliys. J., (Lett), 263, L.79.

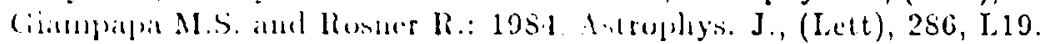

llitimh 13.Al. it "16: 1983, Astropligs, J., 26\%, 280.

Il,iish, B.M.: 1986, Irish Astrmunt. J., 17, 200.

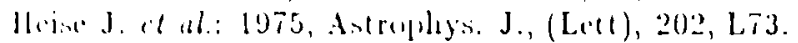

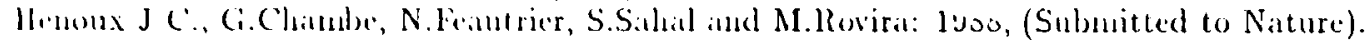

Hjellung, R..11.: 1080, Highlights of Astromony, 5, 857.

limdu M.R., lakison P.D., White S.M ind Meloziz M.: 1987, Astrophys. J., 312, 822.

limulu M.R. and Shevgiontiur R.K.: 1955, Astrophys. J., 297, 6.4.

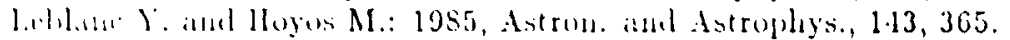

Mathon P.e'.11.: 19s's, Antrophy's. J., (Lett), 330, L131.

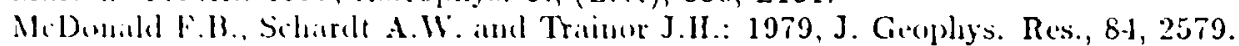

Mullan l.J.: 19it, lrish Astromon. J., 12, 161.

()hamat I'. and Siakii J.: 19ST, Astroplyes. J., 313, 440.

Rumer R. and Vatinat G.S.: 1975, Astropleys. J., 222, 1104.

liwner R., Gulub,I. ambl Valina G S.: 1955, Ann. Rev. Astron and Astropleys., 23, 113.

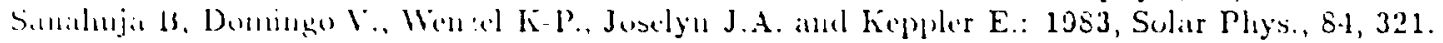

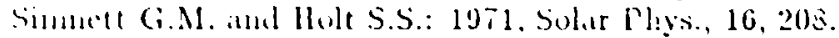

Sinmett G.M1.: 19si, Sular Phiss, 106, 165.

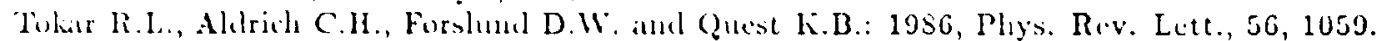

Whito N.E., Cullame J.L., Parmits A.N. and Sweeney M.A.: 10S7, MNRAS, 227, 5.15. 